\title{
La relación entre religión, espiritualidad y salud: una revisión crítica desde las ciencias sociales*
}

\author{
Jean Paul Sarrazin**
}

Artículo de revisión sobre la relación entre los conceptos de religión, espiritualidad y salud presentes en la literatura científica.

Recibido: 26 de junio de 2019

Evaluado: 3 de mayo de 2020

Aceptado: 3 de septiembre de 2020

Publicado: 1 de julio de 2021

Citar como:

Sarrazin, J. P. (2021). La relación entre religión, espiritualidad y salud. Una revisión crítica desde las ciencias sociales. Hallazgos, 18(36), 409-442.

https://doi.org/10.15332/2422409X.5232

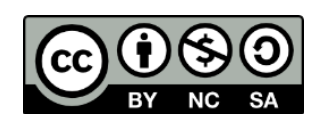

\section{Resumen}

En este artículo se presenta una revisión crítica de la literatura científica sobre los efectos de la espiritualidad y la religiosidad en la salud humana. Mediante un análisis hermenéutico de los textos, se encontró que este tipo de publicaciones carece de definiciones suficientemente claras y consensuales sobre los conceptos y las categorías analíticas. Por esta

\footnotetext{
* Este artículo surge del proyecto de investigación titulado "Espiritualidad y salud. Una perspectiva cualitativa y fenomenológica del caso colombiano", que el autor lleva a cabo de manera independiente. Universidad de Antioquia, Medellín, Colombia.

** Doctor en Sociología por la Université de Poitiers, Francia. Docente-investigador vinculado al Departamento de Sociología en la Universidad de Antioquia, Colombia. Correo electrónico: jean.sarrazin@udea.edu.co

ORCID: https://orcid.org/0000-0002-8022-4674
}

Hallazgos

ISSN: 1794-3841 | e-ISSN: 2422-409X | DOI: https://doi.org/10.15332/2422409X

Vol. 18 N.0 36| julio-diciembre del 2021 
razón, se recurre a los aportes de distintas investigaciones en ciencias sociales sobre los fenómenos religiosos y, especialmente, sobre el advenimiento y la creciente difusión del concepto de espiritualidad en las sociedades occidentales contemporáneas. Se concluye que es necesario un nuevo marco teórico-metodológico que permita dar cuenta de las creencias individualizadas y desinstitucionalizadas propias de la modernidad tardía, y se plantean nuevas hipótesis sobre la relación de causalidad entre espiritualidad y salud. Finalmente, se observa la necesidad de poner en práctica una fenomenología de la espiritualidad, con el fin de conocer los sentidos y las experiencias que las poblaciones locales asocian al concepto de espiritualidad.

Palabras clave: Creencia religiosa; Espiritualidad; Modernización; Práctica religiosa; Salud; Sociología de la religión.

\section{The relationship between religion, spirituality and health: a critical review from the social sciences}

\section{Abstract}

This article presents a critical review of the scientific literature on the effects of spirituality and religiousness on human health. Through a hermeneutical analysis of the texts, it was found that this type of literature lacks sufficiently clear and consensual definitions of the analytical concepts and categories. For this reason, the article resorts to the contributions of different social science research on religious phenomena and, especially, on the advent and growing diffusion of the concept of spirituality in contemporary Western societies. It is concluded that a new theoretical-methodological framework is necessary to account for the individualized and deinstitutionalized beliefs typical of late modernity, and new hypothesis are proposed on the causal relationship between spirituality and health. Finally, the need to implement a phenomenology of spirituality is recommended, in order to improve our understanding of the meanings and experiences that local populations associate with the concept of spirituality. 
Keywords: Religious belief; Spirituality; Modernization; Religious practice; Health; Sociology of religion.

\section{A relação entre religião, espiritualidade e saúde: uma revisão crítica a partir das ciências sociais}

\section{Resumo}

Este artigo apresenta uma revisão crítica da literatura científica sobre os efeitos da espiritualidade e da religiosidade na saúde humana. Por meio de uma análise hermenêutica dos textos, constatou-se que esse tipo de publicação carece de definições suficientemente claras e consensuais dos conceitos e categorias analíticas. Por esse motivo, são utilizadas as contribuições de diferentes pesquisas nas ciências sociais sobre os fenômenos religiosos e, principalmente, sobre o advento e a crescente difusão do conceito de espiritualidade nas sociedades ocidentais contemporâneas. Conclui-se que é necessário um novo marco teóricometodológico para dar conta das crenças individualizadas e desinstitucionalizadas próprias da modernidade tardia, e novas hipóteses sobre a relação causal entre espiritualidade e saúde são propostas. Por fim, observa-se a necessidade de colocar em prática uma fenomenologia da espiritualidade, a fim de conhecer os sentidos e experiências que as populações locais associam ao conceito de espiritualidade.

Palavras-chave: Crença religiosa; Espiritualidade; Modernização; Prática religiosa; Saúde; Sociologia da religião.

\section{Introducción}

La relación entre religión, religiosidad, espiritualidad y salud humana, tanto mental como física, es un tema que ha tomado una gran importancia en las últimas décadas. De acuerdo con Harold G. Koenig (2008), notable especialista en la materia, hay más de 3000 informes originales de investigación científica en los que se ha estudiado empíricamente dicha relación. Con el paso de los años, este tipo de literatura ha crecido de 
forma cada vez más acelerada (McCarroll et ál., 2005). La mayoría de tales estudios presentan una correlación positiva entre la adopción de una religión o espiritualidad y la mejoría en la salud (Koenig et ál., p. 461). Entre estos se hallan numerosos análisis que han mostrado los beneficios de la religión o la espiritualidad en la salud, especialmente cuando se trata de reducir enfermedades relacionadas con el estrés, como la presión alta (Tartaro et ál., 2005) o la depresión (Krause, 2009). Los efectos de la religiosidad sobre la reducción del estrés evidentemente llevan a un mayor bienestar emocional, mental y físico en las personas (Bradshaw y Ellison, 2010; Navas y Villegas, 2007).

Como señalan Quinceno y Vinaccia (2009), en cuanto a la salud física, "los estudios han estado centrados particularmente en la actividad inmunológica, las neoplasias y enfermedades cardiovasculares, cerebrovasculares, neurológicas y dolor" (pp. 327-328); en lo que respecta a la salud mental, "los estudios han estado enfocados en las adicciones, el suicidio, la delincuencia, la ansiedad-depresión, el estrés, la esquizofrenia, el psicoticismo y los trastornos bipolares" (p. 328). En los distintos tipos de trabajos, concluyen los mencionados autores, se "obtiene como resultado un mismo principio: relaciones positivas y causales concluyentes entre espiritualidad y salud" (pp. 8-9).

Otras fuentes reiteran que "la espiritualidad y la religiosidad pueden otorgar mecanismos de afrontamiento de la enfermedad de diversa índole: cognitivos, afectivos, psicológicos y comportamentales" (Puentes Silva et ál., 2015, p. 484). Este tipo de investigaciones se han difundido también en medios masivos de comunicación, como es el caso del periódico $E l$ Colombiano, en el que se anuncia que "en estudios científicos como uno publicado en el Journal of Nursing Scholarship en 2007, la espiritualidad ha mostrado ser efectiva para reducir los niveles de ansiedad, estrés y depresión" (Cortés, 2018). 
La mayoría de los estudios citados sobre esta correlación provienen de la biomedicina y la psicología, y se han basado en rigurosas mediciones, experimentos y estadísticas. Aunque dicha correlación está ya bastante documentada, la investigación que dio origen a este artículo permite constatar la ausencia de claridad en esa gran cantidad de estudios respecto a las características específicas de la religiosidad que incidirían positivamente en el bienestar humano. Por demás, muchas de estas investigaciones toman los conceptos de religión y de espiritualidad de forma indiscriminada, lo cual es problemático. No obstante, se han hecho ciertas claridades, aunque estas se refieren sobre todo al concepto de religión, más que al de espiritualidad. Por ejemplo, Lim y Putnam (2010) observan que la mayoría de estudios sobre religión y salud han encontrado que la razón principal de esta relación de causalidad positiva radica en los vínculos sociales y el apoyo que los sujetos practicantes encuentran en las comunidades o congregaciones religiosas. En otras palabras, las comunidades religiosas brindan a sus miembros un apoyo psicosocial que incide positivamente en su salud.

En esta literatura se asume que ser creyente o practicante de alguna religión o espiritualidad significa pertenecer a una comunidad religiosa específica y claramente identificada (por ejemplo, pertenecer a una comunidad católica, musulmana, evangélica, budista, etc.). Es en el seno de la comunidad religiosa que los individuos encontrarían los vínculos sociales funcionales a su bienestar psíquico y somático. Esta noción de religión, entendida como una "comunidad moral", cohesionada y claramente identificada, corresponde con la definición de religión propuesta desde hace más de un siglo por uno de los clásicos de la sociología, Emile Durkheim (1982): “Una religión es un sistema solidario de creencias y de prácticas relativas a las cosas sagradas, es decir, separadas, interdictas, creencias y prácticas que unen en una misma 
comunidad moral, llamada Iglesia, a todos aquellos que se adhieren a ellas" (p. 42).

Sin embargo, aquella noción de religión desconoce las formas no institucionalizadas de religiosidad (Luckmann, 1973), así como el hecho de que una creciente población en el mundo se considera como "espiritual pero no religiosa" (Cornejo y Blázquez, 2016, p. 12; Frigerio, 2016, p. 209; Woodhead, 2010, p. 37). Como se puede notar al hacer una revisión de la literatura, muchos de estos reportes científicos referidos hablan también de la "espiritualidad" de los actores sociales, y frecuentemente se toma el concepto como si fuera un sinónimo de religión o de religiosidad. Lo anterior es un grave error epistemológico que tiene incidencias en la metodología utilizada a la hora de realizar investigaciones empíricas.

Se encuentran diferentes artículos que se basan en el concepto de espiritualidad como algo distinto de la religión, pero no lo definen de manera suficientemente clara. En efecto, quienes han notado el hecho señalan que el término espiritualidad ha sido objeto de inconsistencias, controversias y confusiones (Koenig, 2012, p. 2; Reinert y Koenig, 2013, p. 2622). Puentes Silva et ál. (2015, p. 484) ratifican que las definiciones de religión, religiosidad y espiritualidad no son del todo claras en la literatura, y que los términos suelen intercambiarse o asumirse como equivalentes.

Si bien se puede suponer que las religiones son aquellas instituciones o comunidades organizadas bajo una denominación específica y explícita, el concepto de espiritualidad denota algo distinto. Aquella relación causal entre una mejor salud y el apoyo que se recibe de comunidades o congregaciones religiosas no sería relevante, entonces, en el caso de las espiritualidades, ya que estas últimas parecen existir principalmente por fuera del marco de una organización, congregación, iglesia o comunidad definida. Si es cierto que, lejos del apoyo comunitario, la espiritualidad 
tiene un efecto subjetivo en el bienestar de los individuos, es necesario especificar las características de dicha categoría, máxime cuando esta se difunde cada vez más en la población (Frigerio, 2016).

Considerando todo lo anterior, este artículo presenta los aportes de las ciencias sociales útiles para entender el concepto de espiritualidad en nuestra modernidad tardía. Esto constituye un fundamento para que nuevas investigaciones científicas (incluyendo psicología, medicina y salud pública) puedan avanzar en la compresión de la correlación positiva que existe entre la espiritualidad y una mejor salud. Con tal fin se muestra la importancia de conocer previamente un contexto sociocultural en el que se desarrollan las nuevas formas del creer, las cuales están influenciadas por procesos macrosocietales propios de la modernidad, tales como la desinstitucionalización y la individualización (Beck, 2009), a los que me referiré más adelante.

\section{La espiritualidad en las terapias alternativas}

La importancia que adquiere la espiritualidad en nuestros días se evidencia en prácticas y creencias relacionadas con la salud y el bienestar de las personas. Esto se hace evidente sobre todo en las terapias alternativas. En efecto, la asociación entre espiritualidad y salud suele ocurrir por fuera de instituciones reconocidas formalmente como médicas o sanitarias (Greira y Urgell, 2002). En Colombia, debido al deficiente servicio que prestan las entidades de salud, se refuerza el rechazo por una institucionalidad biomédica hegemónica, lo que incentiva el recurso a medicinas alternativas en las que se puede encontrar el concepto de espiritualidad.

Las formas de medicina alternativa son muy variadas y dinámicas, y se pueden hallar en lo que se conoce como el "entorno holístico" (Blázquez y Cornejo, 2013). Según la perspectiva holística, la salud humana depende 
de todas las dimensiones que componen su ser, entre las cuales se incluyen la corporal, la mental, la emocional y la espiritual. En este entorno se descubren formas de "pensamiento positivo", "superación personal", nuevas psicoterapias, coaching, bioenergética, etc., al lado de prácticas asociadas a Oriente, como es el caso del yoga, la meditación o el reiki. Este tipo de manifestaciones espirituales y terapéuticas alternativas están cada vez más presentes en las principales ciudades del mundo occidental en general, incluyendo, por supuesto, a Colombia.

Por lo demás, existe una gran cantidad de referencias en los medios masivos de comunicación del país que vinculan la espiritualidad y la salud. Por tomar solo una muestra reciente, veamos algunos ejemplos. La revista Semana, el semanario más importante del país, publica un artículo titulado: “¿Por qué ser espiritual ayuda a vivir mejor?”. Allí se asocia la espiritualidad a la meditación, práctica que supuestamente permite el "bienestar interior" (Semana, 2017). En otro ejemplo, la cadena de radio y televisión Caracol (una de las más importantes del país) tiene una sección titulada "Sanamente", a la cual acuden distintos invitados cuyos perfiles son descritos en el sitio web de la cadena: 1) "Administradora de Empresas de la Universidad Javeriana. Trabajó 15 años en Citibank y lleva 15 años trabajando como terapeuta con ángeles y la espiritualidad”; 2) “Coach espiritual y personal con amplia experiencia. Ha impartido talleres y certificaciones en diferentes lugares del mundo. Es escritor, conferencista y capacitador. Guía a otros en procesos que sanan la mente y dan paz"; 3) "Socióloga y profesora con una amplia experiencia en el campo de la formación de personas que buscan una vida mejor [...] coordinadora nacional de BKWSU (Universidad Espiritual Mundial Brahma Kumaris) en Brasil” y, además, trabaja el tema del "empoderamiento e inclusión desde la espiritualidad"; 4) "Director de Brahma Kumaris Colombia con 34 años de experiencia en Meditación Raja Yoga. Conferencista, coach y autor 
internacional", propone actividades con el fin de "iniciar el año con espiritualidad" (Caracol Radio, 2018).

Una práctica que en Colombia ha venido extendiéndose notoriamente, y en la que se hace explícita la relación entre espiritualidad y salud, es el yoga. Cada vez más difundido en Occidente, a tal punto que es considerado como un fenómeno globalizado (Hoyez, 2005), el yoga es representado en Colombia como una manera de "conectar la mente, el cuerpo y la espiritualidad" (El Tiempo, 2018), y es usual escuchar que se trata de una práctica "espiritual" con efectos positivos en la salud.

Paralelamente al yoga, en Occidente crece el conocimiento sobre la meditación, y sobre ella también se puede encontrar información en medios masivos de comunicación. Así, por ejemplo, en El Tiempo, el diario de mayor difusión en el país, se lee que la meditación generaría "cada vez más interés entre la población general y la comunidad científica" y que "la meditación es generalmente vinculada a la espiritualidad”, y se habría posicionado en el modelo médico occidental "para el tratamiento de problemas físicos y psicológicos" (Londoño, 2018).

Por último, vale la pena mencionar una práctica originaria del continente americano (a diferencia del yoga o la meditación, comúnmente asociadas a Oriente, aunque menos difundida que estas dos), práctica que podemos encontrar en varias ciudades colombianas. Se trata de las "tomas de yagé" o rituales chamánicos en los que un grupo de personas se reúne en torno a un chamán, quien administra dosis de un brebaje con sustancias psicoactivas, entre las cuales se encuentra el extracto de la planta conocida internacionalmente como ayahuasca (Banisteriopsis caapi). Este ritual, cuyas finalidades varían considerablemente dependiendo del sujeto que participe en él, suele ser interpretado como una experiencia espiritual y como una práctica que produce mejorías en la salud (Sarrazin, 2008). Cabe subrayar que quienes asisten a las tomas de yagé y le otorgan ese 
sentido espiritual también pueden frecuentar otro tipo de terapias alternativas, como el yoga, la meditación trascendental, la psicología transpersonal o los temazcales. Tales prácticas suelen ir acompañadas de discursos sobre la espiritualidad y la importancia de una transformación interior del sujeto como condición benéfica para la salud.

En general, las representaciones sociales de prácticas como el yoga, la meditación o las tomas de yagé, en la medida en que se relacionan con la salud (particularmente en su versión alternativa), también son asociadas con frecuencia a la espiritualidad, aunque este último término no suele ser definido con claridad por los actores mismos o por quienes han escrito sobre el tema. Llama la atención que este tipo de prácticas y creencias existen sobre todo entre personas con altos niveles de educación, ubicados en los estratos socioeconómicos medio y alto, y muchos de ellos se consideran agnósticos o ateos (Beltrán, 2012, p. 215; Sarrazin, 2012). "En nuestra sociedad contemporánea cada vez resulta más frecuente encontrar a personas que se consideran ateas y espirituales" (Ramos, 2016, p. 165). No debe, entonces, sorprender que los adeptos a la espiritualidad (y su vínculo con la salud) se declaren críticos de una religión como el catolicismo, el pentecostalismo o el islam.

Todo lo anterior muestra que importantes sectores de la población en Occidente reinterpretan las distintas tradiciones como poseedoras de una sabiduría espiritual que estaría al alcance de todos y que sería fuente de bienestar y salud. Estamos ante un fenómeno cultural transnacional que marca "la adopción generalizada de algunas prácticas religiosas tradicionales como parte de un repertorio espiritual universal, que por añadidura se conciben como instrumentos de autocuidado en salud" (Cornejo y Blázquez, 2013, p. 21, las cursivas son propias). ¿A qué se debe esta asociación? ¿Cuáles son las características de esa "espiritualidad" que llevan a un mayor bienestar? Para comenzar a responder esas preguntas, 
se debe comenzar por conocer el marco general y más amplio en el que aparece el auge actual del concepto de espiritualidad.

\section{El contexto sociocultural en el que surge la espiritualidad moderna}

Se puede comenzar recordando que la teoría de la secularización según la cual la religiosidad iría cediendo terreno a medida que los países se iban modernizando es una teoría que, aunque tuvo mucha fuerza en el siglo XIX y primera mitad del siglo XX, ha sido falseada contundentemente en las últimas décadas. Hoy sabemos que existe un proceso de "dessecularización" del mundo, acompañado de una pluralización de las expresiones religiosas (Berger, 1999). Una mayor visibilización de estas expresiones en el espacio público (Habermas, 2006), aunado a su reconocimiento por parte de la academia, incluyendo las ciencias naturales y médicas, representan unas de las razones por las cuales la literatura sobre la relación entre religiosidad y salud ha aumentado.

En cuanto a la espiritualidad moderna, esta debe ser entendida en el marco de dinámicas culturales y procesos históricos particulares de gran importancia. Estamos ante transformaciones estructurales como son la pérdida de credibilidad en que la ciencia, el desarrollo tecnológico y el progreso permitan a los seres humanos tener mayor bienestar y vivir en un mundo mejor. Al mismo tiempo, se observa el declive de la confianza en algunas instituciones sociales (como las iglesias tradicionales) o las críticas al materialismo y al capitalismo, considerados como responsables de la destrucción medioambiental (Habermas, 2006).

Por otro lado, también es cierto que se ha desmoronado la hegemonía cultural ejercida por la Iglesia católica desde épocas coloniales (Bastian, 2004; Bidegain y Demera, 2005, entre otros), fenómeno que ocurre en el marco de procesos relativamente recientes en la modernidad como la 
expansión del liberalismo económico, político y cultural, el pluralismo, la difusión de una educación crítica de las figuras autoritarias y tradicionales o los flujos de la globalización (Parker, 2005).

Las transformaciones del creer en la actualidad son considerables. "Hoy en día, los principales rasgos del fenómeno religioso son la inestabilidad, la emergencia constante de nuevas formas de organización y acción, su carácter híbrido y el sincretismo en los cultos y creencias" (Tejeiro, 2007, p. 39). Es así que la secularización no significa más que una disminución del poder de las religiones en cuanto formas institucionalizadas y tradicionales del creer (Casanova, 2009). La multiplicación de formas inéditas y emancipadas de la institucionalidad tradicional (católica o protestante) es una tendencia propia de modernidad tardía: la “desinstitucionalización” (Beck, 2009). Parte de este fenómeno se manifiesta de manera notable, aunque muy poco estudiada, en un aumento de personas que se autodefinen mediante afirmaciones como "creo en Dios pero no en la religión”, "creo en Dios a mi manera" (Beltrán, 2012, p. 209), o quienes dicen ser adeptos a las "espiritualidades alternativas" o creyentes en su "propia espiritualidad" (Sarrazin, 2017a; Urrea y Castrillón, 2000).

Hay un "creer sin pertenecer" (Davie, 1990) o un creer independiente de la pertenencia duradera a una comunidad religiosa tradicional (Willaime, 1996). Por la misma vía, se observa en América Latina "la adscripción religiosa que ya no enmarca ni delimita las dinámicas creyentes de los individuos" (De La Torre, 2012, p. 218). En el curso de sus vidas, los sujetos se pueden adherir a diferentes tipos de creencias y prácticas, e incluso mostrar una múltiple adhesión en un mismo momento (King y Hedges, 2014).

Se observan, entonces, expresiones altamente inestables y móviles, con muy bajos niveles de regulación y estructuración para denominar prácticas 
y creencias, por ejemplo, "new age" en Colombia, en las que es evidente una valoración del concepto de espiritualidad (Sarrazin, 2012). Así, aunque se haya constatado una disminución de los asistentes a las iglesias católicas, crecen estas nuevas formas de espiritualidad que no obedecen a dogmas institucionalizados, en las que los individuos reclaman independencia para escoger en qué creer y qué practicar.

Las instituciones religiosas tradicionales, por supuesto, no han dejado de existir, pero muchos individuos recurren a ellas alternativamente y sin suscribirse a sus lógicas de manera definitiva. El sujeto que se autodenomina "espiritual" usa y transforma las tradiciones existentes (Van der Veer, 2009, p. 1109). Se ha observado que estas nuevas formas del creer siguen una modalidad "a la carta" (De La Torre, 2012; Couture, 2000; Hervieu-Léger, 1999; Parker, 2005). Esto significa que los sujetos parecen escoger "a su gusto" sus creencias y prácticas dentro de una amplia gama de posibilidades, y combinan elementos de diversas culturas, religiones y tradiciones a manera de bricolage (Hervieu-Léger, 1999) ${ }^{1}$, con el fin de incrementar su bienestar.

\section{Hacia una caracterización de la espiritualidad en la modernidad}

Es de lamentar, como lo hacen Houtman y Aupers (2007, p. 308), el gran déficit de estudios empíricos y sistemáticos sobre las visiones de mundo presentes en la religiosidad desinstitucionalizada o por fuera de iglesias; aquello que Luckmann (1973) llamará la "religión invisible", y de la cual forman parte las expresiones de espiritualidad. Asimismo, es lamentable la escasez de estudios empíricos sobre la construcción social del concepto de espiritualidad en la contemporaneidad (Houtman y Aupers, 2007, p. 317).

\footnotetext{
${ }^{1}$ El término bricolage ha sido tomado del francés y, en particular, de la obra de Claude Lévi-Strauss (2009).
} 
Y es que, como señala Van der Veer (2009) - uno de los pocos investigadores prominentes en el mundo que ha asumido este reto desde el punto de vista histórico-, esta espiritualidad moderna (y, por lo tanto, propia de una era de secularización) es particularmente difícil de definir (2009, p. 1097).

En un esfuerzo por tipificar las definiciones de espiritualidad que se presentan en la literatura científica relacionada con temas de salud, se pueden identificar ocho tipos de definiciones: 1) el sentido y propósito de la vida; 2) conexiones y relaciones; 3) Dios(es) y un Otro trascendente; 4) un Yo trascendente; 5) un principio vital; 6) una fuerza unificadora o una energía integradora; 7) creencias exclusivamente privadas y personales; 8) la esperanza (McCarroll et ál., 2005, pp. 45-53). Cabe anotar que estas nociones y acercamientos al concepto de espiritualidad se han encontrado en poblaciones moderno-occidentales contemporáneas, es decir que en otros contextos sociohistóricos o en otras culturas lo que se entiende por espiritualidad puede variar considerablemente.

Para Houtman y Aupers (2007), se trata de una "espiritualidad postcristiana" (post-Christian spirituality). Esta categoría, según los autores, se relaciona con creencias como la existencia de un espíritu o fuerza vital, la reencarnación o la vida después de la muerte o un dios personal, todo lo cual se acompaña de un rechazo a los dogmas eclesiales.

Esta pluralidad de definiciones de espiritualidad impide tomar una sola de estas a manera de categoría analítica utilizable en investigaciones empíricas de forma sistemática. Cada una simplemente corresponde con algunos de los posibles sentidos que tiene la palabra para ciertos sectores poblacionales. Tomar solo uno de esos sentidos como el único válido negaría una diversidad de creencias que es importante visibilizar. Tampoco nos corresponde adoptar una posición normativa que pretenda asegurar cuál es el "verdadero" sentido del concepto. 
Por un lado, sería por demás errado seguir una tendencia en la literatura académica en la que se asume que la espiritualidad es "una suerte de impulso universal y ahistórico en el ser humano" (Cornejo y Blázquez, 2016, p. 12). Esta perspectiva universalizante y acrítica debe ser superada. Es la modernidad, luego del siglo XIX, la que imagina que la espiritualidad es una categoría universal y que podría, por lo tanto, estar presente en cualquier sociedad o tradición del planeta (Van der Veer, 2009, p. 1100). Por otro, tampoco podemos limitarnos a la presunción según la cual la espiritualidad englobaría solo las creencias en espíritus, ya que este no es necesariamente el sentido que por lo general se le da al concepto en la modernidad tardía (Van der Veer, 2009). Recuérdese, por ejemplo, la asociación que algunas personas hacen entre espiritualidad y la esperanza o las conexiones.

Comúnmente se asume que las religiones pueden contener elementos de espiritualidad, pero la espiritualidad no estaría restringida a las religiones tradicionales. Por otro lado, la espiritualidad parece tener sentido por oposición a lo material y está asociada vagamente a nociones como la moral, el psiquismo, el bienestar, la salvación, la liberación, lo sagrado, lo trascendental, lo místico y lo sobrenatural (Koenig, 2012, p. 3). Todo ello, sin embargo, no es suficientemente preciso, ya que estos últimos términos tampoco son definidos con claridad en la literatura; tienen sentidos muy divergentes, y algunos de ellos pueden asimismo ser asociados a la religión ("lo sagrado" o "lo sobrenatural") o a lo secular (como "la moral" o "la liberación”).

Existen, por supuesto, razones históricas que explican parcialmente la asociación entre espiritualidad y religión: no se debe perder de vista que la espiritualidad como tal es un concepto cristiano (Van der Veer, 2009), es decir, que nace en el marco religioso de Occidente. Sin embargo, con el avance de la modernidad y en un proceso que comienza en el siglo XIX, los 
conceptos empiezan a disociarse: en algunas ocasiones se considera que la espiritualidad es un concepto más amplio que el de religión, ya que la primera está presente en las diferentes religiones, pero no necesariamente implica la pertenencia a grupos organizados o la adhesión a denominaciones de fe específicas (O’Connor et ál., 2005, p. 2). En realidad, se ha observado que gran parte de los elementos que definen la religión y la espiritualidad se traslapan (Koenig, 2012). En ciertos casos, se asume que todas las religiones tienen un componente espiritual, y en otros casos, que la espiritualidad puede estar en las religiones y también por fuera de ellas, por lo cual la categoría de lo espiritual sería más abarcadora que la de lo religioso (Quiceno y Vinaccia, 2009, p. 325).

Investigaciones en ciencias sociales han mostrado que durante la modernidad muchos de los elementos antiguamente considerados como "religiosos" se han ido transformando y han adquirido nuevas etiquetas por fuera del lenguaje de las religiones institucionalizadas. Según Carrete y King (2005), muchas de las expresiones culturales que antes eran conocidas como "religiosas", desde los años 1990 empiezan a considerarse como "espirituales". Es así que muchos contenidos religiosos se han venido actualizando y se expresan a través de lenguajes como los de "la energía, la filosofía positiva, la ecología, el vegetarianismo y el crecimiento personal” (Semán y Viotti, 2015, p. 87). Así mismo, se constata que esos contenidos se encuentran ahora dispersos en prácticas que se conocen como "espirituales" o "terapéuticas" (Bourdieu, 2000; Flanagan y Jupp, 2007). De esta manera, las investigaciones empíricas de las complejas realidades sociales demuestran la dificultad de trazar una tajante separación analítica y operacional entre lo religioso y otro tipo de manifestaciones culturales (Sarrazin, 2019) ${ }^{2}$.

\footnotetext{
${ }^{2}$ Al analizar la división entre la religión y la medicina (división instaurada en la Ilustración), Bourdieu (1971) muestra que esto se debe más a rivalidades entre
} 
Y es que desde las ciencias sociales se sabe que las religiones son en realidad sistemas culturales (Geertz, 2003) que inciden de manera general en el comportamiento y el pensamiento de las poblaciones, proporcionando ontologías (Eliade, 1996) y sentido, haciendo inteligible el mundo mediante una serie de categorías de la percepción (Berger, 1971), fundamentando motivaciones duraderas y formulando concepciones de un orden general de la existencia (Geertz, 2003, p. 89). Así mismo, la religión desempeña funciones normativas que señalan los comportamientos moralmente correctos o incorrectos (Durkheim, 1982). Por otra parte, los preceptos religiosos pueden estar tan profundamente relacionados con los ideales de purificación y de sanidad, que los unos a veces se hacen indistinguibles de los otros (Douglas, 1973; Sarrazin y Tamayo, 2017).

Es por eso por lo que si en un momento dado los dioses mueren, escribiera Durkheim (1982, p. 398), la situación de incertidumbre no podría durar eternamente: siempre vuelven a surgir nuevos ideales y figuras sagradas que guían al menos por un tiempo a la humanidad. Aunque vivamos en una "era secular", como Charles Taylor lo plantea, los sujetos no han dejado de recurrir a "marcos de trascendencia" (Taylor, 2007), entre los cuales la espiritualidad es una de las modalidades más recientes. Los individuos que ya no se adhieren a las iglesias tradicionales pueden recurrir a las referencias "espirituales", las cuales se convierten en las nuevas fuentes normativas, identitarias, motivacionales y, sobre todo, de sentido, con lo que se cumplen funciones que antes eran satisfechas por las religiones institucionalizadas.

especialistas (los nuevos médicos y las tradicionales autoridades religiosas) que a diferencias objetivas y observables en las realidades sociales.

Hallazgos

ISSN: 1794-3841 | e-ISSN: 2422-409X | DOI: https://doi.org/10.15332/2422409X

Vol. 18 N.0 36| julio-diciembre del 2021 


\section{La espiritualidad como alternativa}

Las investigaciones empíricas sobre el concepto de espiritualidad en Occidente demuestran que el auge de este fenómeno se enmarca en una tendencia moderno-tardía de rechazo a las religiones instituidas y aliadas al poder estatal (Cornejo, 2012; Van der Veer, 2009). Así, un número creciente de personas en Occidente ahora rechazan las "religiones" y prefieren declararse adeptos a la espiritualidad (Aupers y Houtman, 2010; Díez de Velasco, 2000, p. 23; Heelas y Woodhead, 2005). En el imaginario social, la palabra religión se suele asociar a organizaciones e instituciones cuyos dogmas, autoridades y normas restringen la libertad de los individuos.

La espiritualidad, en cambio, estaría más acorde con los intereses personales más profundos y las inquietudes existenciales, sin obligar al sujeto a pertenecer a una organización en particular o a seguir una autoridad externa (Aupers y Houtman, 2010; Greira y Urgell, 2002; Woodhead, 2010). En nuestra modernidad, "se estableció una diferencia entre religión (más centrada en el dogma) y espiritualidad (más centrada en la experiencia personal)" (Ramos, 2016, p. 169). Esta diferenciación, sin embargo, nada tiene de natural o universal, puesto que surge en un sistema cultural particular y es producto de un proceso sociohistórico de Occidente. Este proceso implica el "descrédito de las religiones institucionalizadas entendidas como organismos de poder, asfixiantes o incluso corruptos" (Ramos, 2016, p. 168). La espiritualidad es, entonces, valorada de forma positiva en la medida en que se imagina como una construcción completamente independiente de toda institución y, por lo tanto, inmune a todo tipo de corrupción o abuso de poder.

Además de tomar sentido por oposición al concepto de religión, como acabamos de ver, el concepto de espiritualidad también es concebido como una forma de oposición o resistencia frente al racionalismo de la 
Ilustración (Heelas y Woodhead, 2005, p. 30). Paradójicamente, no obstante, también existe una convergencia entre racionalismo y espiritualidad (Cornejo y Blázquez, 2016). Semejante paradoja se explica porque la espiritualidad se representa como el conjunto de creencias o sensibilidades de quien, desde una autoproclamada racionalidad propia, es capaz de criticar las iglesias al igual que la razón hegemónica eurocéntrica, patriarcal, burguesa.

Por otro lado, la valoración de la espiritualidad apela también a quienes denuncian una modernidad excesivamente secularista y materialista que no proporciona fuentes de sentido a la existencia (Habermas, 2006, p. 244). En síntesis, la espiritualidad en Occidente se convierte en un dispositivo de protesta contra distintas estructuras hegemónicas, ya sean las iglesias tradicionales, el racionalismo, el secularismo o el materialismo capitalista. Esta connotación contrahegemónica del concepto de espiritualidad es compartida en cierta medida con los distintos tipos de terapias alternativas a los que hemos hecho referencia, ya que estos últimos mantienen una posición de marginalidad y crítica frente a la biomedicina hegemónica.

Una característica de las corrientes espiritualistas - por utilizar la categoría propuesta por Hervieu-Léger y Davie (2010, p. 228) - es que no constituyen organizaciones claramente delimitables bajo una autoridad única. Al contrario, conforman redes más o menos informales y móviles, siguiendo así una tendencia observada en el conjunto de las sociedades modernas y gracias al desarrollo de nuevas tecnologías de comunicación y transporte. En esta modernidad "líquida" (Bauman, 2002), los vínculos humanos se debilitan, las comunidades duraderas se hacen menos probables y la sociabilidad no depende necesariamente de las relaciones cara a cara. Las redes que se configuran en las espiritualidades de tipo new age han sido estudiadas, entre otros autores, por Wood (2007) y 
Woodhead (2010), quienes identificaron formas de autoridad temporales y descentralizadas. Allí se observa igualmente la importancia que los sujetos atribuyen a las experiencias personales y a la autonomía (Carozzi, 1999).

Este fenómeno moderno-tardío lo resume Frigerio (2016) así:

Para sus defensores, la palabra "espiritualidad" permite entender y visibilizar adecuadamente a una forma contemporánea y cada vez más creciente de religiosidad que enfatiza tanto la experiencia religiosa subjetiva como una actitud frente a la fuente legítima de autoridad religiosa que prescinde de, o desenfatiza, el rol de los grupos religiosos tradicionales (principalmente iglesias) colocando al individuo como árbitro último de su validez. (p. 209)

La centralidad del yo observada en estas "corrientes espiritualistas" es un fenómeno relativamente reciente. "Fue en los años 1970 que se empieza apenas a desarrollar la idea de una espiritualidad como auto-realización [sic] y, por lo tanto, un enfoque centrado en el individuo" (Cornejo y Blázquez, 2016, p. 25). Lo anterior implica también que el individuo pretende no seguir una autoridad externa a él, sino ser él mismo su propia autoridad (Aupers y Houtman, 2010, p. 139; Wood, 2007, p. 29). En la llamada espiritualidad subjetiva o self-spirituality, "el individuo comparece como autoridad última de su cosmología y sus creencias al tiempo que se guía por sus emociones y sensaciones a la hora de encontrarse con lo sagrado" (Cornejo et ál., 2014, p. 237).

En este escenario, los diferentes agentes prestadores de algún tipo de servicio espiritual entran a competir en lo que ha sido denominado un “mercado religioso" (Berger y Luckmann, 1997). El pluralismo religioso, promovido por buena parte de los Estados latinoamericanos (incluyendo el colombiano), ha favorecido tal mercado. Siguiendo las lógicas neoliberales, se promueve la libertad de oferta y demanda cultural. Los proveedores de ese mercado (profesores de yoga, de meditación, chamanes, coaches, etc.) 
buscan formas de atraer la demanda a través de sofisticados discursos, puestas en escena, referencias a identidades tradicionales, etc. (Gutiérrez, 2008). De este modo se propician experiencias y emociones positivas para el individuo moderno.

Los sujetos creyentes, por su parte, pueden ser entendidos como consumidores de bienes y servicios espirituales (Berger y Luckmann, 1997; Possamai, 2002). Las religiones tradicionales vienen a ser percibidas como "reservorios de signos" y "materiales simbólicos eminentemente maleables" (Hervieu-Léger, 1999) que pueden ser reciclados por los individuos en función de sus intereses. La espiritualidad se representa como un contenido cultural que estaría presente en las distintas tradiciones religiosas del mundo. Cualquier persona, desde esa perspectiva, podría aprender lecciones de espiritualidad de cualquiera de esas tradiciones y usarlas para incrementar su bienestar. Este fenómeno ocurre gracias a lo que Giddens llama un proceso de deslocalización (disembedding) de las prácticas y creencias, las cuales son extraídas de sus contextos locales de interacción social, para luego ser reestructuradas en contextos socioculturales nuevos (Giddens, 1994, p. 30).

La emancipación de los individuos con respecto a formas institucionales de religión, para reclamar la libertad individual de consumir espiritualidades, escogiendo de manera supuestamente autónoma sus creencias y prácticas, debe entenderse en el marco del proceso de “individualización” (Beck, 2009). Este proceso, cada vez más marcado en la modernidad (Beck, 2009), trae consigo un sujeto que valora su autonomía y, en consecuencia, pretende basarse en sus propios intereses y criterios para tomar decisiones y construir su subjetividad. La espiritualidad contemporánea refleja claramente esa tendencia. En palabras de Cornejo y Blázquez (2016), esta espiritualidad "pivota sobre la constitución del sujeto moderno occidental y las ideologías individualistas 
que lo relatan" (p. 14). Este individualismo es incompatible con la institucionalidad religiosa tradicional, ya que esta última implica el seguimiento de reglas y autoridades que violarían el imperativo de autonomía.

Cabe notar, no obstante, que dicha autonomía es más un ideal moderno que una realidad fáctica (Foucault, 1991), ya que las decisiones de cualquier individuo están influenciadas necesariamente por las estructuras de pensamiento y de lenguaje que no surgen del mismo individuo, sino del medio social en el que este se formó. En otras palabras, es importante tener en cuenta la construcción sociocultural de las subjetividades. Hecha esta aclaración, el ideal de autonomía hace que sea muy baja la probabilidad de que los sujetos modernos adeptos a la espiritualidad conformen organizaciones suficientemente estables y cohesionadas, prefiriendo la constitución de grupos pequeños y de corta duración.

El ideal de autonomía que se impone en estos medios no debe hacernos caer en el error - en cuanto investigadores - de creer que la espiritualidad es en efecto un fenómeno completamente atomizado. Gracias a etnografías realizadas en entornos donde se presenta la espiritualidad alternativa (Wood, 2007; Aupers y Houtman, 2007; Sarrazin, 2012), se han identificado patrones claramente colectivos que, además, están conectados con procesos sociales a escalas micro, meso y macro.

Los procesos de individualización y desinstitucionalización de las sociedades modernas no significan, de ninguna manera, que los individuos existan como entes verdaderamente autónomos, independientes en su totalidad de las influencias del entramado social en el que viven. La búsqueda de libertad individual y autonomía es un fenómeno colectivo precisamente porque se han convertido en un imperativo cultural (Bauman, 2002). Esos individuos "libres" y "autónomos" siguen patrones 
de consumo muy similares e integrados a redes transnacionales de creencias y mercancías "alternativas” (Sarrazin, 2017b).

Por otro lado, no podemos olvidar que una característica general de los nuevos movimientos espiritualistas es su constante referencia a la salud (Hervieu-Léger y Davie, 2010, p. 237). En esa medida, la espiritualidad puede entenderse como un dispositivo constituyente de lo que Foucault (1996) denomina las "tecnologías del yo", es decir, prácticas y procedimientos (incluyendo, por ejemplo, formas de meditación o de alimentación) que los sujetos incorporan a su cotidianidad y mediante las cuales buscan la transformación de sí mismos, según determinados parámetros de lo que es un cuerpo "bello", "saludable", "puro", "elevado", etc. $\mathrm{Y}$ así como en los medios espiritualistas es constante la referencia a la salud, también hay que notar que las terapias alternativas (cada vez más masificadas), empleando la referencia frecuente a la espiritualidad, contribuyen a difundir el interés por esta.

La literatura antropológica muestra que, en diversas sociedades, las religiosidades suelen estar profundamente conectadas con creencias y prácticas relativas al tratamiento de enfermedades (Cornejo y Blázquez, 2016, p. 2). Por eso no debe sorprender que el advenimiento de la espiritualidad contemporánea lleve a un cambio en la manera en que los sujetos modernos se relacionan con su cuerpo y entienden la salud (Giordan, 2009). Mientras que en el catolicismo tradicional (e incluso en el cartesianismo y en el pensamiento ilustrado de la modernidad temprana) se hace una separación tajante entre el cuerpo y el espíritu, en los medios espiritualistas toma fuerza la idea de una conexión profunda de esas dos dimensiones del ser (Giordan, 2009). Así, se habla de una "espiritualidad holista” (Woodhead, 2010, p. 38), según la cual se asume que el ser humano es un "todo" (whole) que incluye diferentes dimensiones interrelacionadas como el cuerpo, la mente, las emociones y 
el espíritu. Esto implica que las acciones que se tomen a favor de una dimensión incidirán en las otras dimensiones y en el bienestar general del sujeto.

\section{Conclusión y recomendaciones}

Una revisión de la literatura sobre la relación entre espiritualidad y salud muestra que no existe claridad ni consenso sobre el significado del primer concepto. Por esta razón, este importante campo de investigaciones científicas (especialmente de las ciencias de la salud) carece de suficiente rigurosidad. Dicha falencia puede solventarse mediante la investigación empírica desde las ciencias sociales, con miras a contribuir a la comprensión del sentido de este concepto de espiritualidad en las sociedades contemporáneas. Así pues, es necesario escuchar a los sujetos mismos, y paralelamente ir conociendo las prácticas, emociones o estados que son asociados al concepto en cuestión.

Esto demuestra la pertinencia de una colaboración interdisciplinaria más estrecha entre las ciencias sociales (historia de las ideas, sociología de la religión, antropología cultural, etc.) y las ciencias de la salud. Un enfoque construccionista (desde el cual se entiende que todo concepto es una construcción social) permitirá aclarar los términos, y en esa medida se establecerán con mayor certeza las correlaciones entre las distintas variables.

Desde hace solo unas décadas, las ciencias sociales han aportado al proceso de caracterización de la espiritualidad como fenómeno cultural propio de la modernidad tardía, lo que nos permite, entre otras cosas, enmarcarlo en procesos macrosocietales, tales como la desinstitucionalización y la individualización. Ambos procesos tienden a desgastar la hegemonía antes ejercida por las religiones tradicionales y organizadas, cuyas autoridades fijas y centralizadas ejercían un control 
sobre los individuos, y en torno a las cuales se estructuraban comunidades relativamente cohesionadas y duraderas.

La espiritualidad, en cambio, se presenta como un conjunto de valores íntimos, creencias personales y prácticas que surgirían de los gustos y orientaciones de un individuo supuestamente autónomo. Aunque dicha autonomía debe ser vista de forma crítica - pues ignora la construcción social de las subjetividades -, la verdad es que este ideal de un sujeto autónomo limita las probabilidades de que se formen comunidades de apoyo psicosocial fuertes y numerosas de modo suficiente. Son otros los mecanismos que incidirían en la salud de los medios espiritualistas, los cuales requieren ser estudiados de manera mucho más cuidadosa, es decir, procurando siempre una vigilancia epistemológica respecto a las categorías analíticas empleadas.

Con base en todo lo anterior, el presente estudio permite plantear una serie de hipótesis que constituyen puntos de partida para futuras investigaciones empíricas:

1. A pesar de ser el producto de procesos de desinstitucionalización e individualización, esto que ahora se conoce como "espiritualidad" (y que se diferencia de "religión”) obedece a ciertas creencias y prácticas institucionalizadas que no son individuales sino colectivas, como la creencia en una realidad trascendente, superior, que está más allá de las vicisitudes del mundo "material" en el que vivimos. Creer en esta trascendencia le permite al individuo relativizar los males que pueda estar padeciendo o las circunstancias que actualmente lo agobian, procedentes de este mundo "material" (recordemos que la espiritualidad se distingue, por oposición, de la materialidad). Tal relativización, sin duda, posibilita disminuir los niveles de estrés que causan o agravan enfermedades. 
2. La vida individual se concibe como un camino de aprendizaje para acercarse a esa realidad trascendente; por lo tanto, la espiritualidad es un recurso en dicho camino. En este sentido, las enfermedades se interpretan como oportunidades de aprendizaje y, en cierta manera, ejemplos de por qué debemos desligarnos emocionalmente del mundo material para acercarnos a lo que es superior y más importante. Esto le permite al individuo enfermo atribuir un sentido positivo a la enfermedad, generándole así una mejor disposición psíquica y emocional, que incide positivamente en su salud.

3. Existe en el medio espiritualista la creencia en una relación estrecha entre el espíritu, la mente y el cuerpo. Aunque se cree que el espíritu y el cuerpo son dimensiones distintas del ser, ambas están conectadas, vinculadas, interrelacionadas, de manera que la vivencia del cuerpo incide en el espíritu, y viceversa. Esto hace que buena parte de los individuos se preocupen no solo por cultivar su espiritualidad, sino también por mantener su cuerpo en un estado óptimo. El cuerpo se enaltece tanto como la espiritualidad, y se convierte en un vehículo hacia aquella dimensión superior. Al respecto, hemos notado que los espiritualistas sacralizan el yo, el cual es, en parte, el cuerpo. No está de más decir que ese ser sagrado debe recibir los más esmerados cuidados en salud.

4. Si bien es cierto que el medio espiritualista se caracteriza por su individualización y se diferencia de la religión justamente por la ausencia de lazos comunitarios fuertes, es importante recordar que en este también existen comunidades, aunque son incipientes, esporádicas, de muy corta duración, y sus lazos interpersonales son más débiles; la reciprocidad y mutua ayuda es menor. De cualquier manera, estas comunidades espiritualistas pueden generar - al menos mínimamente- condiciones psicosociales favorables para la salud (tal 
como se ha observado en los practicantes de religiones institucionalizadas). Cabe añadir que las comunidades espirituales pueden ser virtuales en dos sentidos de la palabra: el contacto entre individuos se realiza únicamente a través de nuevas tecnologías de comunicación o las comunidades son solamente imaginadas por los sujetos, como cuando se cree que pertenecemos a una comunidad mundial unida en torno a una causa espiritual, o cuando se habla de una conciencia universal que nos permite sentirnos unidos a la naturaleza o al universo entero. Este tipo comunidades virtuales puede generar en los actores una cierta sensación de pertenencia y unión, emociones positivas que inciden en su salud.

Desde el punto de vista metodológico, se recomienda recurrir a observaciones etnográficas y al análisis de las narrativas de los actores sociales directamente involucrados, puesto que es necesario estudiar el sentido, las prácticas y las experiencias tal y como son entendidas y vividas por las personas. Será pertinente plantear acercamientos fenomenológicos (Berger y Luckmann, 1966) y, más específicamente, una "fenomenología de la espiritualidad", según como lo proponen Cassaniti y Luhrmann (2014, p. 342). Mediante esta propuesta se busca comprender los procesos a través de los cuales los sujetos asocian ciertos eventos mentales o corporales al concepto de espiritualidad.

\section{Sobre el autor}

Jean Paul Sarrazin. Antropólogo con Opción en Filosofía de la Universidad de los Andes, Colombia. Máster (suma cum laude) en Migraciones y Relaciones Interétnicas de la Université de Poitiers, Francia. Doctor (suma cum laude) en Sociología de la Université de Poitiers, Francia. Profesor Asociado del Departamento de Sociología de la Universidad de Antioquia, Colombia. Áreas de interés: sociología y 
antropología de las religiones, estudios culturales, análisis del discurso y de las representaciones sociales. Publicaciones recientes: "Definiciones del concepto de 'religión' en el marco de las relaciones de poder modernas" (2021); “La espiritualización del objeto arqueológico: análisis de las narrativas contemporáneas sobre lo indígena y la diversidad en dos museos colombianos" (2020); "Lo Sagrado en la Política: cuestionando la teoría de la diferenciación de las esferas" (2020); "Elementos para una crítica de las políticas dirigidas a la protección de la diversidad cultural en Colombia" (2019); "Religión: ¿sabemos de lo que estamos hablando? Examen sobre la viabilidad de una categoría analítica para las ciencias sociales" (2018).

\section{Referencias}

Aupers, S. y Houtman, D. (2010). The social and public significance of new age spirituality. En S. Aupers y D. Houtman (Eds.), Religions of modernity: Relocating the sacred to the self and the digital (pp. 135-160). Brill Academic Publishers.

Bastian, J. P. (2004). La recomposición religiosa de América Latina en la Modernidad Tardía. La modernidad religiosa: Europa latina y América Latina en perspectiva comparada (pp. 155-174). Fondo de Cultura Económica.

Bauman, Z. (2002). Modernidad líquida. Fondo de Cultura Económica.

Beck, U. (2009). El Dios personal: la individualización de la religión y el "espíritu" del cosmopolitismo. Paidós.

Beltrán, W. (2012). Descripción cuantitativa de la pluralización religiosa en Colombia. Universitas Humanística, (73), 201-237.

Berger, P. (1971). El dosel sagrado: elementos para una sociología de la religión. Amorrortu.

Berger, P. (Ed.) (1999). The desecularization of the world: resurgent religion and world politics. William Berdmans Publishing.

Berger, P. y Luckmann, T. (1966). The social construction of reality: A treatise in the sociology of knowledge. Anchor Books. 
Berger, P. y Luckmann, T. (1997). Modernidad, pluralismo y crisis de sentido. Paidós.

Bidegain, A. M. y Demera, J. D. (2005). Globalización y diversidad religiosa en Colombia. Universidad Nacional de Colombia.

Bourdieu, P. (1971). Genèse et structure du champ religieux. Revue Française de Sociologie, 12(3), 295-334.

Bourdieu, P. (2000). Cosas dichas. Gedisa.

Bradshaw, M. y Ellison, C. (2010). Financial hardship and psychological distress: Exploring the buffering effects of religion. Social Science \& Medicine, 71(1), 196204.

Caracol Radio. (2018, 22 de marzo). Sanamente.

http://caracol.com.co/programa/2018/05/26/sanamente/1527291295 359427.ht $\underline{\mathrm{ml}}$

Carozzi, M. (1999). La autonomía como religión: la nueva era. Alteridades, 9(18), 19-38.

Carrette, J. y King, R. (2005). Selling spirituality: The silent takeover of religion. Routledge.

Casanova, J. (2009). The secular and secularisms. Social Research, 76(4), 1049-1066.

Cassaniti, J. y Luhrmann, T. (2014). The cultural kindling of spiritual experiences. Current Anthropology, 55(S10), 333-343.

Cornejo, M. (2012). Religión y espiritualidad: ¿dos modelos enfrentados? Trayectorias post-católicas entre budistas Soka Gakkai. Revista Internacional de Sociología, $70(2), 327-346$.

Cornejo, M. y Blázquez, M. (2013). La convergencia de salud y espiritualidad en la sociedad postsecular. Las terapias alternativas y la constitución del ambiente holístico. Revista de Antropología Experimental, 13(2), 11-30.

Cornejo, M. y Blázquez, M. (2016). De la mortificación a la New Age: genealogía y política de las espiritualidades terapéuticas contemporáneas. Nómadas. Revista Crítica de Ciencias Sociales y Jurídicas, (46), 1-26. http://dx.doi.org/10.5209/NOMA.53537

Cornejo, M., Blázquez, M. y Flores, J. (2014). El reencuentro de la salud y la espiritualidad: agencias saberes y prácticas periféricas. Periferias, Fronteras y Diálogos. Una Lectura Antropológica de los Retos de la Sociedad Actual (pp. 235241). Publicaciones de la Universitat Rovira i Virgili.

\section{Hallazgos}

ISSN: 1794-3841 | e-ISSN: 2422-409X | DOI: https://doi.org/10.15332/2422409X 
Cortés, H. (2018, 11 de febrero). De la cúrcuma al amor, ¿contra el cáncer todo sirve? El Colombiano. http://www.elcolombiano.com/tendencias/tratamientoscomplementarios-para-el-cancer-curan-XC8172044

Couture, A. (2000). La tradition et la rencontre de l'Autre. En F. Lenoir y Y. TardanMasquelier, Encyclopédie des religions. Vol. 2 (pp. 1373-1400). Vallart.

Davie, G. (1990). Believing without belonging: is this the future of religion in Britain? Social Compass, 37(4), 455-469.

Díez de Velasco, F. (2000). Las nuevas religiones. El Orto.

Douglas, M. (1973). Pureza y peligro: un análisis de los conceptos de contaminación y tabú. Siglo XXI.

Durkheim, É. (1982). Las formas elementales de la vida religiosa. Akal.

De La Torre, R. (2012). Religiosidades nómadas. Ciesas.

Eliade, M. (1996). Lo sagrado y lo profano. Labor.

El Tiempo (2018, 30 de marzo). Nuevo estudio analiza los efectos del tan popular Bikram Yoga. http://www.eltiempo.com/vida/salud/los-efectos-del-bikram-yoga-173924

Flanagan, K. y Jupp, P. (Eds.) (2007). A sociology of spirituality. Ashgate Publishing Limited.

Foucault, M. (1991). Sujeto y poder. Ediciones Carpe Diem.

Foucault, M. (1996). Tecnologías del Yo. Paidós.

Frigerio, A. (2016). La ¿ “nueva”? espiritualidad: ontología, epistemología y sociología de un concepto controvertido. Ciencias Sociales y Religión/Ciências Sociais e Religião, 18(24), 209-231.

Geertz, C. (2003). La interpretación de las culturas. Gedisa.

Giddens, A. (1994). Les conséquences de la modernité. L’Harmattan.

Giordan, G. (2009). The Body between religion and spirituality. Social Compass, 56(2), 226-236. DOI: $\underline{\text { https://doi.org/10.1177/0037768609103356 }}$

Griera, M. y Urgell, F. (2002). Consumiendo religión: un análisis del consumo de productos con connotaciones espirituales entre la población juvenil. Fundación La Caixa. 
Gutiérrez, C. (2008). La danza neotradicional como oferta espiritual en la estantería exotérica New Age. En K. Argyriadis, R. de la Torre, C. Gutiérrez y A. Aguilar (Coords.), Raíces en movimiento: prácticas religiosas tradicionales en contextos translocales (pp. 363-392). El Colegio de Jalisco-Ird-Cemca-Ciesas-Iteso.

Habermas, J. (2006). Entre naturalismo y religión. Paidós.

Heelas, P. y Woodhead, L. (2005). The spiritual revolution: Why religion is giving way to spirituality. Blackwell.

Hervieu-Léger, D. (1999). Le pèlerin et le converti: la religion en mouvement. Flammarion.

Hervieu-Léger, D y Davie, G. (2010). El despliegue espiritual de los nuevos movimientos religiosos. En D. Gutiérrez Martínez (Coord.), Religiosidades y creencias contemporáneas: diversidad de lo simbólico en el mundo actual (pp. 215-240). El Colegio Mexiquense.

Houtman, D. y Aupers, S. (2007). The spiritual turn and the decline of tradition: The spread of post-christian spirituality in 14 western countries, 1981-2000. Journal for the Scientific Study of Religion, 46(3), 305-320. DOI: https://doi.org/10.1111/j.1468-5906.2007.00360.x

Hoyez, A.-C. (2005). L'Espace-monde du Yoga: une géographie sociale et culturelle de la mondialisation des paysages thérapeutiques [Tesis de doctorado]. Université de Rouen. https://tel.archives-ouvertes.fr/tel-00011237v2/document

King, A. y Hedges, P. (2014). What is religion? Or what is it we're talking about? En P. Hedges (Ed.), Controversies in contemporary religion. Vol. 1 (pp. 1-30). Praeger.

Koenig, H. (2008). Concerns about measuring "spirituality" in research. The Journal of Nervous and Mental Disease, 196(5), 349-355.

Koenig, H. (2012). Religion, spirituality, and health: The research and clinical implications. ISRN Psychiatry, 2012(2012), 1-33.

Koenig, H., King, D. y Carson, V. (2012). Handbook of religion and health. Oxford University Press.

Krause, N. (2009). Religious involvement, gratitude, and change in depressive symptoms over time. The International Journal for the Psychology of Religion, 19(3), 155172. DOI: https://doi.org/10.1080/10508610902880204 
Lévi-Strauss, C. (2009). El pensamiento salvaje. Fondo de Cultura Económica.

Lim, C. y Putnam, R. (2010). Religion, social networks, and life satisfaction. American Sociological Review, 75(6), 914-933. DOI: https://doi.org/10.1177/0003122410386686

Londoño, C. A. (2018, 20 de enero). La meditación: un camino hacia la sanación física y espiritual. El Tiempo. http://www.eltiempo.com/vida/salud/beneficios-de-lameditacion-para-la-salud-173360

Luckmann, T. (1973). La religión invisible. Sígueme.

McCarroll, P., O’Connor, T., Meakes, E., Meier, A., O’Connor, T. S. J. y VanKatwyk, P. L. (2005). Assessing plurality in spirituality definitions. En A. Meier, T. S. J. O’Connor y P. L. VanKatwyk (Eds.), Spirituality and health: multi-disciplinary explorations (pp. 43-59). Wilfrid Laurier University Press.

Navas, C. y Villegas, H. (2007). Espiritualidad y salud. Saberes Compartidos, 1, 37-42.

O'Connor, T. y Meakes, E. (2005). Towards a joint paradigm reconciling faith and research. En A. Meier, T. S. J. O’Connor y P. L. VanKatwyk (Eds.), Spirituality and health: Multidisciplinary explorations (pp. 11-22). Wilfrid Laurier University Press.

Parker, C. (2005). ¿América Latina ya no es católica? Pluralismo cultural y religioso creciente. América Latina Hoy, (41), 35-56.

Possamai, A. (2002). Cultural consumption of history and popular culture in alternative spiritualities. Journal of Consumer Culture, 2(2), 97-218.

Puentes Silva, Y., Urrego Barbosa, S. y Sánchez Pedraza, R. (2015). Espiritualidad, religiosidad y enfermedad: una mirada desde mujeres con cáncer de mama. Avances en Psicología Latinoamericana, 33(3), 481-495. https://doi.org/10.12804/revistas.urosario.edu.co/apl/a.3226

Quiceno, M. y Vinaccia, S. (2009). La salud en el marco de la psicología de la religión y la espiritualidad. Diversitas: Perspectivas en Psicología, 5(2), 321-336.

Ramos, A. (2016). Ateísmo y espiritualidad. 'Tlu. Revista de Ciencias de las Religiones, (21), 165-183. https://doi.org/10.5209/ILUR.53851 
Reinert, K. G. y Koenig, H. G. (2013). Re-examining definitions of spirituality in nursing research. Journal of Advanced Nursing, 69(12), 2622-2634. DOI:

https://doi.org/10.1111/jan.12152

Sarrazin, J. P. (2008). El chamanismo es un camino: las culturas indígenas como fuentes de sabiduría espiritual en Bogotá. En A. Aguilar et ál. (Eds.), Raíces en movimiento: prácticas religiosas tradicionales en contextos translocales (pp. 329362). El Colegio de Jalisco-Ciesas.

Sarrazin, J. P. (2012). New Age en Colombia y la búsqueda de espiritualidad indígena. Revista Colombiana de Antropología, 48(2), 139-162.

Sarrazin, J. P. (2017a). ¿Guiados por Dios o por sí mismos? Estudio comparativo entre adeptos a las espiritualidades alternativas y adeptos a las iglesias evangélicas. Cuestiones Teológicas, 44(102), 373-396.

Sarrazin, J. P. (2017b). Budismo universal, budismo individual: análisis del interés por la espiritualidad oriental en Occidente. Escritos, 25(54), 59-81. DOI:

https://doi.org/10.18566/escr.v25n54.a04

Sarrazin, J. P. (2019). Religión: ¿̇sabemos de lo que estamos hablando? Examen sobre la viabilidad de una categoría analítica para las ciencias sociales. Criterio Libre, 16(29), 67-84.

Sarrazin, J. P. y Tamayo, N. (2017). Vivir islámicamente en Colombia: manifestaciones de una búsqueda de trascendencia. Nómadas, (46), 65-79.

Semán, P. y Viotti, N. (2015). “El paraíso está dentro de nosotros”. La espiritualidad de la Nueva Era, ayer y hoy. Nueva Sociedad, (260), 81-94.

Semana. (2017, 15 de agosto). ¿Por qué ser espiritual ayuda a vivir mejor? https://www.semana.com/vida-moderna/articulo/beneficios-y-problemas-de-lareligion/539213

Tartaro, J., Luecken, L. J. y Gunn, H. (2005). Exploring heart and soul: Effects of religiosity/spirituality and gender on blood pressure and cortisol stress responses. Journal of Health Psychology, 10(6), 753-66. DOI:

https://doi.org/10.1177/1359105305057311

Tejeiro, C. (2007). La investigación social del fenómeno religioso en Colombia. En C.

Tejeiro, F. Sanabria y W. Beltrán (Eds.), Creer y poder, hoy (pp. 29-59).

Universidad Nacional de Colombia.

Hallazgos

ISSN: 1794-3841 | e-ISSN: 2422-409X | DOI: https://doi.org/10.15332/2422409X

Vol. 18 N.o 36| julio-diciembre del 2021 
Taylor, C. (2007). A secular age. Harvard University Press.

Urrea, F. y Castrillón, M. (2000). Religiosidades fundamentalistas y alternativas en procesos de globalización. Revista de Estudios Sociales, (5), 73-84.

Van der Veer, P. (2009). Spirituality in modern society. Social research: An international quarterly, 76(4), 1097-1120.

Willaime, J. P. (1996). Dinámica religiosa y modernidad. En G. Giménez (Coord.), Identidades sociales y religiosas en México (pp. 47-65). Instituto Francés de América Latina-Instituto de Investigaciones Sociales-UNAM.

Wood, M. (2007). Possession, power, and the New Age: Ambiguities of authority in neoliberal societies. Ashgate.

Woodhead, L. (2010). Real religion and fuzzy spirituality? Taking sides in the sociology of religion. En A. Stef y H. Dick (Eds.), Religions of modernity: relocating the sacred to the self and the digital (pp. 31-48). Brill Academic Publishers. 